\title{
High-resolution neutron time-of-flight measurements for light water at the Spallation Neutron Source (SNS), Oak Ridge National Laboratory
}

\author{
Luiz Leal ${ }^{1, *}$,Vaibhav Jaiswal ${ }^{1}$, and Alexander I. Kolesnikov ${ }^{2}$ \\ ${ }^{1}$ Institut de Radioprotection et de Sûreté Nucléaire PSN-EXP/SNC/LNR, Fontenay-aux-Roses, 92260, France \\ ${ }^{2}$ Neutron Scattering Sciences Division, Oak Ridge National Laboratory, Oak Ridge, TN 37831-6473, USA
}

\begin{abstract}
Series of light water inelastic neutron scattering experiments have been made at the Oak Ridge National Laboratory (ORNL), Spallation Neutron Source (SNS) covering temperatures ranging from $295 \mathrm{~K}$ to $600 \mathrm{~K}$ and pressures of 1 bar and 150 bar. The temperatures and pressures ranges correspond to that of pressurized light water reactors. The inelastic scattering measurements will help the development of light water thermal scattering kernels, also known as $S(\alpha, \beta)$ thermal scattering law (TSL), in a consistent fashion given the amount and the quality of the measured data. Light water thermal scattering evaluations available in existing nuclear data libraries have certain limitations and pitfalls. This paper introduces the state of the art of the light water thermal scattering cross-section data not only for room temperature but as well as for reactor operating temperatures, i.e. 550 - $600 \mathrm{~K}$. During the past few years there has been a renewed interest in re-investigating the existing TSL models and utilize the recent experimental data or perform molecular dynamics simulations. It should be pointed out that no single TSL evaluation is based entirely on experimental data and one has to rely on TSL models or a combination of both. New TOF measurement of light water at the SNS, with a detailed description of the experimental setup, measurement conditions, and the associated foreseen results is presented in this paper. The analysis of the experimental data would help in validating the existing approach based on old experimental data or based on molecular dynamic simulations using classical water models, knowledge of which is very important to generate TSL libraries at reactor operating conditions.
\end{abstract}

\section{Introduction}

Light water is the most widely used moderator in thermal nuclear reactors, such as pressurized water reactors (PWRs). The cross-sections and the distribution of neutrons in the thermal energy range are determined by the interatomic binding of the hydrogen atoms in the molecular system [1]. In the standard thermal scattering libraries, these effects are described by a $S(\alpha, \beta)$ function, which is often termed as thermal scattering law (TSL) [2]. Until recently, standard TSL data libraries have relied mostly on experimental data measured in the 60s [3] and are based on physics models $[1,2]$. During the past few years, there has been a renewed interest in reviewing these models and in generating new thermal scattering libraries based on the recent advancement in the time-of-flight (TOF) experiments and improved computational capabilities [4-8]. A promising technique which provides reliable TSL involves Molecular Dynamic (MD) simulations of light water. This method allows the computation of TSL using MD trajectories but is not completely model-free, due to the intrinsic limitation of the classical MD simulations. The accuracy of these simulations also depends on the water potentials used at high temperature and pressure (reactor normal operating conditions) [9]. Experimental TSL for high temperature and pressure are very scarce. Consequently, one

*e-mail: luiz.leal@irsn.fr still observes some discrepancies between the evaluation and benchmark results at reactor normal operating conditions, even with the most recent TSL data libraries.

New experimental TSL data would facilitate the validation of MD simulations results and the applicability of water potentials at high temperature and pressure. High-resolution double-differential cross-section (DDXS) of light water at high temperature and pressure is of interest for the reactor physics community. In addition, quantification of experimental uncertainties, both the systematic and statistical will play a very important role in generating the data covariance and will help in cross-section validation.

\section{Need for new measurements}

TSL evaluations for light water have evolved in years, and the most recent ones differ based on the different methodologies adopted for their generation. TSL for light water available in the ENDF/B-VIII.0 is based on MD simulations and the other in JEFF-3.3 is based on TOF experiments. Also, they both differ significantly, especially concerning the temperature dependence and the way of characterization of the diffusion properties of the light water molecule. However, the former effect is more prominent in the thermal energy range and hence more sensitive to thermal reactors. These differences and lack of clear under- 


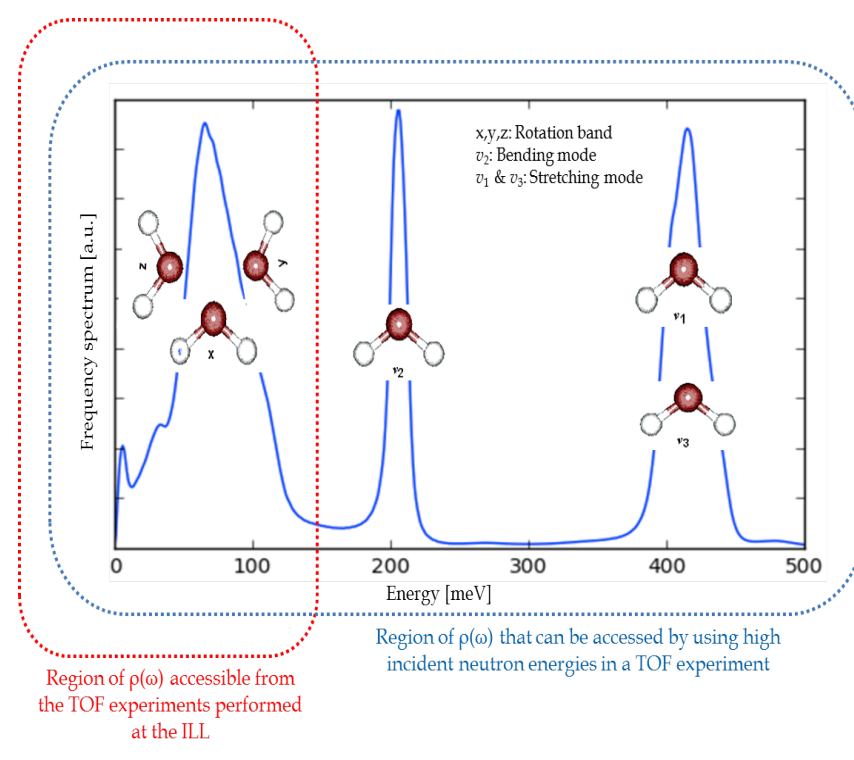

Figure 1. Pictorial diagram representing the different energy bands in the frequency spectrum that can be accessible depending on the choice of incident neutron energy. The data for this particular frequency spectrum has been taken from Ref. [6].

standing of the temperature behavior of the TSL presents a need of new TOF experiments to better understand the physics behind thermal scattering law for light water to help prepare new TSL evaluations, if not at least help validating the existing evaluations. These new experiments would serve two purposes:

- To generate new TSL libraries fully based on the experimentally measured frequency spectrum. However, this route is challenging and requires a thorough data reduction procedure.

- To validate the existing TSLs experimentally that are based on theoretical models and or molecular dynamic simulations.

The experimental approach adopted at IRSN to generate TSL was started by performing TOF experiments with light water at the ILL using the IN4c and IN6 spectrometers. The TSL libraries generated during this work based on the TOF data, i.e., $S(\alpha, \beta)_{\text {ILL }}$ was derived using the measured frequency spectrum [10]. However, the frequency spectrum obtained from both the IN4c and IN6 spectra can only reveal information about the rotational band due to the chosen incident energy of the neutron, which limits the energy transfer range. Due to this limitation the high energy modes, i.e., the bending and the stretching modes were not accessible in the measured spectra, and these modes were approximated using harmonic oscillators while preparing the LEAPR parameters to generate the TSL. This approximation works quite well but poses a challenge to define the energy of these oscillators, which is not well known experimentally for high temperatures. To avoid these approximations, using the full frequency spectrum of light water extending to higher energy transfers would be more reliable. The range of the frequency spectrum accessible at the ILL experiment and the one required to generate full TSL is shown in Fig. 1.

Experimental measurement of the TSL is quite challenging and needs careful correction to obtain reliable data. Reason being the measured data has to be corrected for multiple scattering effects, background and sample holder corrections, measurement times, detector normalization, etc. Due to these inherent limitations the scientific community either resolves to the experimentally derived frequency spectrum or MD simulations to obtain either directly the TSL or the frequency spectrum to be later used by the LEAPR module of the NJOY code [11]. However, the differential or double differential data acts as an excellent tool for the verification and validation of the physics models of the TSLs involved. Comparison of the TSLs with the differential and double differential data could indicate the limitations/failures of a particular model and hence indicate improvements.

Overall, a new TOF experiment would help us improve our knowledge about the dynamic structural properties of light water. This knowledge would help us better model the thermal scattering law or help us improve the present TSL evaluations. The idea behind this paper is not to present a new TSL for light water, instead to have better insights about the physics involved with the dynamic structure factor of water and hence the derived frequency spectrum. Almost all the TSLs presently available is benchmarked using room temperature critical benchmarks. Due to the lack of experimental benchmark data at high temperature (reactor operating conditions), it poses a significant challenge to validate or have confidence in the hightemperature TSLs.

There is still a need for high-resolution differential and total cross-section data for light water at high temperatures and pressures on a broad energy and momentum transfer range. Figure 1 represents a pictorial sketch of the frequency spectrum that can be accessed depending on the choice of incident neutron energies. As it can be seen, the entire frequency spectrum is accessible by choosing a high incident neutron energy, which makes facilities like the Spallation Neutron Source (SNS) at the Oak Ridge National Laboratory (ORNL), a favorable option to carry out TOF experiments. Also, high-resolution measurements of energy and momentum transfers are required to study the dynamical structure properties of light water with a satisfactory signal to noise ratio.

\section{SEQUOIA TOF Spectrometer}

Considering the requirement of high resolution inelastic neutron scattering measurement, it appears that the SEQUOIA spectrometer at the SNS is one of the best-suited facilities to study the dynamical structure properties of light water at high temperature and pressure with a satisfactory signal to noise ratio. SEQUOIA allows inelastic neutron scattering studies with fine three-dimensional momentum and energy transfer measurements. Also, the availability of different high incident energies by choosing the appropriate Fermi chopper speed makes it a favorable 
machine for our experiment. The choice of incident energy available to perform the experiments on SEQUOIA lies between 4 to $6000 \mathrm{meV}$. There are around $900{ }^{3} \mathrm{He}$ detector tubes that covers a scattering angle of $-30^{\circ}$ to $60^{\circ}$ horizontally and $-18^{\circ}$ to $60^{\circ}$ vertically. Moderator to Fermi chopper distance is about $18 \mathrm{~m}$ whereas Fermi chopper to sample distance is about $2 \mathrm{~m}$. The sample to detector distance is $5.5-6.3 \mathrm{~m}$ (cylindrical geometry). The energy resolution is between $1-5 \%$ of the incident neutron energy. A pictorial diagram of the SEQUOIA spectrometer is given in Fig. 2.

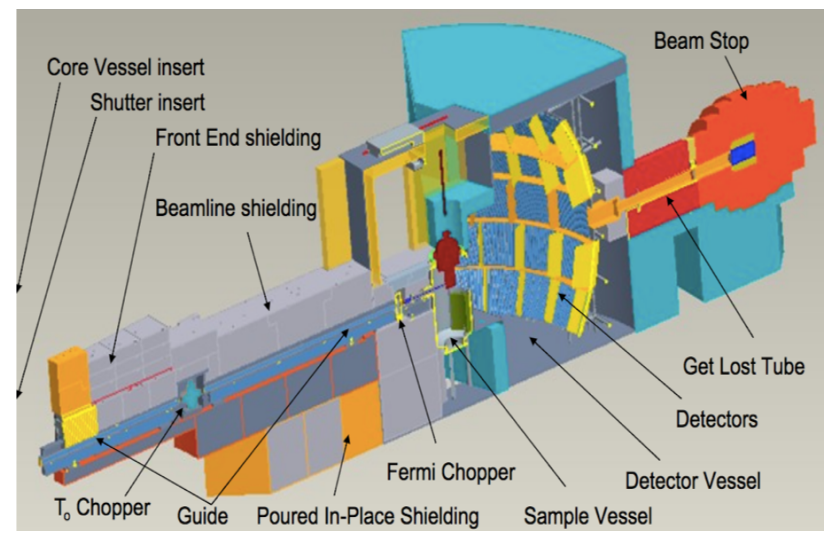

Figure 2. Pictorial diagram of the SEQUOIA spectrometer.

The sample holder/cell that has been used for this experiment is made up of titanium slab with dimensions $50 \times 50 \mathrm{~mm}^{2}$ that covers the entire incident beam. The cell contains $2 \mathrm{~mm}$ thick, cylindrical channels of $1 \mathrm{~mm}$ diameter going from top to the bottom. A schematic diagram of the cell is shown in Fig. 3. The cell is designed in a way that it can withstand temperature up to $823 \mathrm{~K}$ and Pressure up to 250 bar. This temperature and pressure tolerance makes this cell suitable for the present experiment. Also, at the chosen incident energies of the neutron, significant multiple scattering effects are not expected.

\section{Experimental conditions of the measurements}

New DDXS measurements on light water at normal operating conditions of PWRs have been performed in a wide range of energy transfer by using the SEQUOIA spectrometer. The experiment intended to carry out a series of experiments starting at $295 \mathrm{~K}$ (room temperature) up to to $600 \mathrm{~K}$ (reactor operating temperature). The choice behind choosing different incident energies of neutrons to perform the TOF experiment is to explore/investigate different features of the phonon spectrum at different energy transfers. Nine different sets of measurements at five different incident neutron energies help to explore distinct frequency spectrum regions of light water, with an optimum energy and momentum transfer resolution. The neutron energies for which the experiments were performed are: a) 8/12 $\mathrm{meV}$ to study the first acoustic peak around $9 \mathrm{meV}$; b) 60 $\mathrm{meV}$, the translational vibrations of water molecules in the energy range 0 to $40 \mathrm{meV}$; c) $160 \mathrm{meV}$, the range of the rotational band around $50 \mathrm{meV}$ to $140 \mathrm{meV}$; d) $280 \mathrm{meV}$, to resolve the bending of the $\mathrm{H}-\mathrm{O}-\mathrm{H}$ mode around $205 \mathrm{meV}$; and e) $800 \mathrm{meV}$, the stretching of the $\mathrm{O}-\mathrm{H}$ modes around $410 \mathrm{meV}$. Three different sets of measurements were carried out, one with the cell containing light water, one with the empty cell and one with a Vanadium sample that helps in normalization of the data. The thermodynamic conditions of the TOF experiment are shown in Table 1.

Table 1. Thermodynamic conditions of the measurements for light water

\begin{tabular}{llll}
\hline Sample & $\begin{array}{l}\text { Temperature } \\
(\mathrm{K})\end{array}$ & $\begin{array}{l}\text { Pressure } \\
\text { (bar) }\end{array}$ & $\begin{array}{l}\text { Incident neutron } \\
\text { energy }(\mathrm{meV})\end{array}$ \\
\hline \multirow{4}{*}{ Water } & 295 & 1 & $60,160,280,800$ \\
+ & 323 & 1 & $60,160,280,800$ \\
Cell & 350 & 1 & $12,60,160,280,800$ \\
& 400 & 150 & $8,60,160,280,800$ \\
& 450 & 150 & $8,60,160,280,800$ \\
& 500 & 150 & $8,60,160,280,800$ \\
& 550 & 150 & $8,60,160,280,800$ \\
Cell & 600 & 150 & $8,60,160,280,800$ \\
& 295,350, & & \\
& 400,450, & 1 & $8,60,160,280,800$ \\
\hline Vanadium & 550,600 & & \\
\hline
\end{tabular}

The range of incident neutron energies and temperatures allows one to investigate the frequency spectrum leading to accurate $S(q, \omega)$ description. However, at this point, we do not aim at combining the data set measured at different energies to obtain one full frequency spectrum. The data resolution changes with the choice of incident neutron energy and adding or slicing a particular data to obtain a full set is challenging and is a matter of future research. The detailed data analysis is presented in the following paper [12] of the same conference.

\section{Conclusions}

The Institut de Radioprotection et de Sûreté Nucléaire (IRSN), which provides technical support for the French nuclear safety authority, is working on the improvement of nuclear cross-section data, in particular, TSL for light water. Calculations performed using existing TSL for light water, available in most of the nuclear data libraries, show significant differences. The new SNS experimental data will serve as the basis for solving the issues with the existing data. The outcome of this study will also lead to a better interpretation of the physics inherent in the light water, in particular, the vibrational modes of the frequency spectrum and the double differential cross sections. It is envisioned the generation of new TSL evaluations for light water, based on experimentally measured frequency spectrum following validations with experimental critical benchmarks. The accurate measurements carried out at SNS will lead to a better interpretation of criticality safety results and calculations. Also, the evaluation of the new 

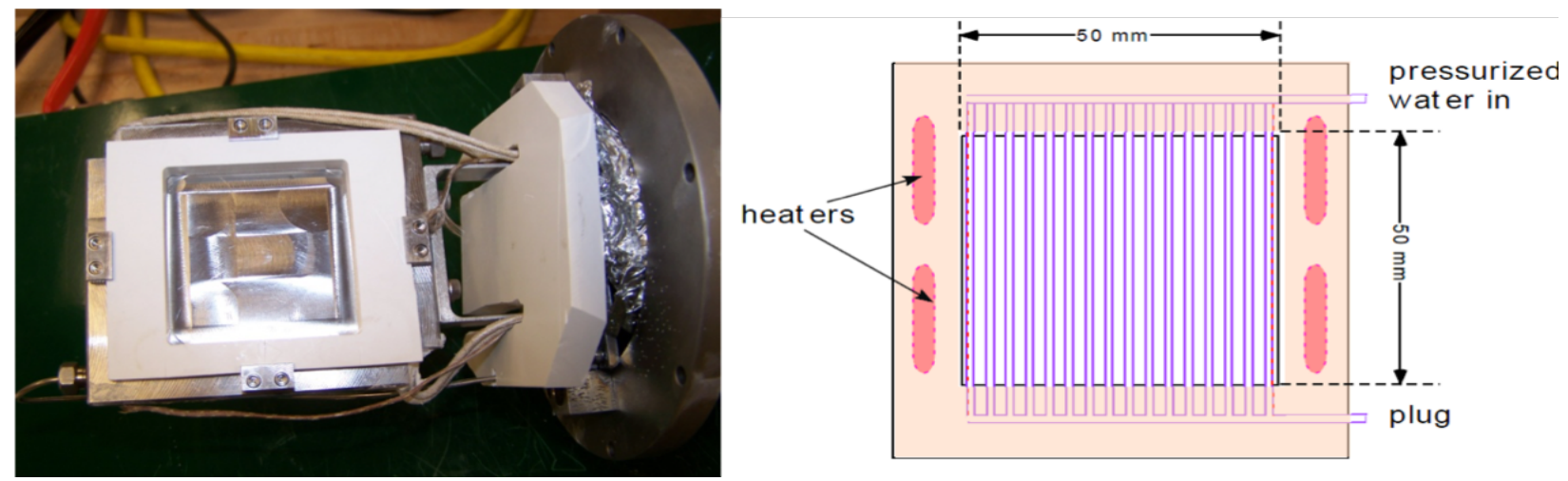

Figure 3. Pictorial diagram (left) and the schematics (right) of the sample holder.

SNS data would provide the grounds to obtain the uncertainties associated with the experimental conditions in connection with the measurements, namely, the systematic and the statistical uncertainties. While existing nuclear data libraries contains TSL evaluation for water for certain PWR operational condition, it should be noted that no uncertainty information exists based on actual experimental data.

\section{Acknowledgements}

This research used resources at the Spallation Neutron Source, a DOE Office of Science User Facility operated by the Oak Ridge National Laboratory. The authors are grateful to Matthew Stone from SNS for his valuable contribution to the INS data measurement and data analysis. The authors would also like to thank Jesse Holmes for fruitful discussions on the evaluation of TSL data based on TOF experiments.

\section{References}

[1] L. V. Hove, "Correlations in Space and Time and Born Approximation Scattering in Systems of Interacting Particles", Physics Review, vol. 95, no. 1, pp. 249-262 (1954).

[2] R. MacFarlane, "New Thermal Neutron Scattering Files for ENDF/B-VI, Release 2" LA-12639-MS (ENDF-356) March (1994).

[3] B. Haywood, I. Thorson, "The Scattering Law for Light and Heavy Water at $20 \mathrm{C}$ and $150 \mathrm{C"}$ " In: Proceedings of the Brookhaven Conference on Neutron thermalization, IAEA (1962).

[4] M. Mattes, J. Keinert, "Thermal Neutron Scattering Data for the Moderator Materials $\mathrm{H}_{2} \mathrm{O}, \mathrm{D}_{2} \mathrm{O}$ and $\mathrm{ZrH}_{x}$ in ENDF-6 Format and as ACE Library for
MCNP(X) Codes" INDC(NDS)-0470, International Nuclear Data Committee (2005).

[5] E. Farhi et al, "Light and heavy water dynamic structure factor for neutron transport codes", Journal of Nuclear Science and Technology, vol. 52, no. 6 (2014).

[6] J. Marquez Damian, J. Granada and D. Malaspina, "CAB models for water: A new evaluation of the thermal neutron scattering laws for light and heavy water in ENDF- 6 format", Annals of Nuclear Energy, vol. 65, pp. 280-289 (2014).

[7] Y. Abe and S. Tasaki, "Molecular dynamics analysis of incoherent neutron scattering from light water via the Van Hove space-time self-correlation function with a new quantum correction", Annals of $\mathrm{Nu}$ clear Engineering, vol. 83, pp. 302- 308 (2015).

[8] V. Jaiswal et. al., "Measurement of double differential cross-section of light water at high temperature and pressure to generate $S(\alpha, \beta)$ ", EPJ Web of Conferences, 146,13006 (2017).

[9] I. Shvab and Richard J. Sadus, "Atomistic water models: Aqueous thermodynamic properties from ambient to supercritical conditions", Fluid Phase Equilibria, Vol. 407, 7-30 (2016).

[10] V. Jaiswal, "Theoretical and experimental approach towards generation of thermal scattering law for light water", Ph.D. thesis, University of Lille, (2018).

[11] R. MacFarlane, D. Muir, "The NJOY Nuclear Data Processing System" LA-12470-M, Los Alamos National Laboratory, USA (1994).

[12] V. Jaiswal, L. Leal, A. I. Kolesnikov, "Analysis of the time-of-flight neutron scattering cross-section data for light water measured at the SEQUOIA spectrometer, Spallation Neutron Source (SNS)", ND2019 conference, Beijing, China, (2019). 